\title{
Morphological Characterization of Ordered Meso-Pores on a Mesoporous Silica by using High-Resolution Scanning Electron Microscopy
}

\author{
Yoshihisa Ohrai,* Takeshi Sunaoshi,* Hiroyuki Ito,* Takeshi Ogashiwa, ${ }^{*}$ Nobuaki Ikawa, ${ }^{* *}$ \\ Akira Endo** \\ * Hitachi High-Technologies Corp., 11-1, Ishikawa, Hitachinaka, Ibaraki, 312-0057, Japan \\ **Research Institute for Innovation in Sustainable Chemistry, National Institute of Advanced \\ Industrial Science and Technology (AIST), AIST Central 5, 1-1-1, Higashi, Tsukuba, Ibaraki, $305-$ \\ 8565, Japan \\ E-mail:orai-yoshihisa@naka.hitachi-hitec.com
}

The field emission scanning electron microscope (FE-SEM) is a powerful tool for observing the fine structure of surfaces at high resolution and is used in various fields, such as nano-materials and soft materials. Mesoporous silicas can have mesostructures such as the two-dimensional (2D) hexagonal type and three-dimensional (3D) cage type. We previously observed the surface structure of 2D hexagonal type mesoporous silicas, which is important in catalytic performance by using FESEM [1][2]. On the other hand, to evaluate the surfaces of single mesoporous particles from various tilt angles is important for 3D cage type mesoporous silicas. However, it is difficult to do this at high resolution, because when a specimen is tilted the working distance become long and the observation conditions, such as the deceleration technique, become restricted. In this study, we used low-kV inlens FE-SEM to observe the surfaces of single mesoporous particles from various tilt angles.

A primary advantage of in-lens FE-SEM is that it is possible to maintain a high-resolution imaging at longer working distances and while the sample is tilted, even up to tilt angles of 40 degrees. Also, the in-lens FE-SEM has a specimen-rotation holder that can rotate specimens 360 degrees. We evaluated the surface structures of single particles of 3D cage-type mesoporous silica (SBA-16) that were produced under various conditions and had a body-centered cubic lattice structure. The specimen-rotation holder was used to adjust the target surface to vertical to suit the direction of the primary electrons. To obtain secondary electron (SE) signals from the outermost surface of the mesoporous silica, we observed the surface at an accelerating voltage of about $1 \mathrm{kV}$.

We obtained SE images of SBA-16 at various magnifications and sample orientations (tilt angle and rotation). Fig. 1 (a)(b)(c) are images of an SBA-16 particle observed from various tilt angles, showing that the SBA-16 particle consists of 12 hexagonal faces and six tetragonal faces. Fig. 1 (d) and (e) are higher magnification images of Fig.1 (b) and (c), respectively.In Fig. 1 (d), the mesopores from the hexagonal face are apparent as a diamond shape with a ratio of diagonals of 1.0:1.4.

In Fig. 1 (e), the mesopores of the tetragonal have a square-shaped structure. These results indicate that the hexagonal faces are in the (110) plane of the body-centered cubic lattice structure and the tetragonal faces are in the (100) plane of the body-centerd cubic structure. The Millar indices obtained for these two structures were the indices expected for this type of mesoporous silica (SBA16). From these results, it was confirmed that high-resolution imaging by in-lens type FE-SEM with the capability of flexible tilting and rotation is valid for evaluating the ultra-surface structures of mesoporous silicas. 
References

[1] N. Ikawa, et al., Chem. Commun., 46 (2010) 4342.

[2] A. Endo et al., Colloids Surf., A, 357 (2010) 11.
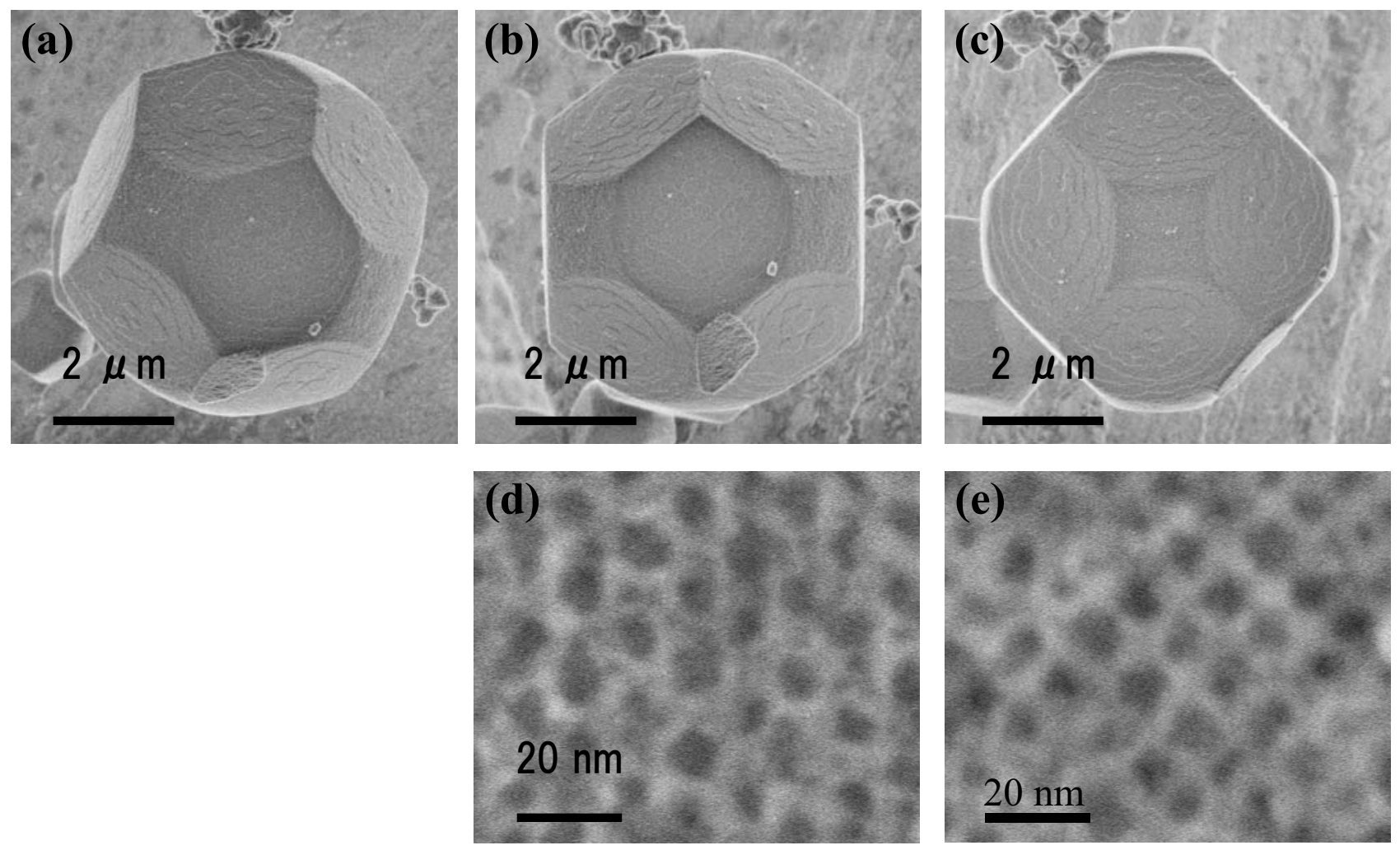

Fig.1 Secondary electron images of SBA-16 (accelerating voltage $1.2 \mathrm{kV}$ )

(a) Image of an SBA-16 particle at a magnification of 10000 times. (b) Image of an SBA-16 particle adjusted to show the hexagonal face. (c) Image of an SBA-16 particle adjusted to show the tetragonal face. (d) Higher magnification of Fig. 1 (b). (e) Higher magnification of Fig. 1 (c). 